\title{
Numerical Study of One Prey-Two Predator Model Considering Food Addition and Anti-Predator Defense
}

\author{
Dian Savitri* \\ Mathematics Depertement, Universitas Negeri Surabaya
}

\begin{abstract}
This article examines the interaction between prey populations, juvenile predators, and adult predators. A mathematical model that considers adding food and anti-predators was developed. The equilibria of the existing system are that the system has four equilibria points with conditions suitable for the locale. Numerical simulations were carried out to describe the dynamics of the system solution. Based on numerical simulations, the varying of parameter causes changes in the extinction of prey or survival of prey populations, juvenile predators, and adult predators. Addfood parameters $(A)$ encourae Hopf Bifurcation and Saddle-node bifurcation Numerical continuity results show that Hopf bifurcation occurs when the parameter value $A=1.00162435$ and when the parameter value $A=2.435303$ Saddle-node bifurcation occurs.
\end{abstract}

Keywords: Mathematic Model, Simulation, Equilibria

\section{Introduction}

The phenomena in ecology, especially in agriculture are interesting to study. Pests are plant-disturbing organisms ranging from roots, stems, and leaves. Planthonppers or insects are one of the pests for plants that can damage crops. Therefore, it is necessary to improve pest control effort that are environmentally friendly. Dragonflies can be used by farmers to control various pests, especially in rice fields. Dragonflies are natural enemies of planthoppers. An adult dragonfly can consume hundreds of leafhoppers every day [1]. Dragonfly juvenile that lives in water prey on leafhoppers or small insects. Adult dragonflies will eat on the other pests on the surface of the water ranging from leafhoppers to insects. The phenomenon of natural enemies can affect the development of pest populations, thereby protecting crops from damage and crop failure. The reduction in the number of pests makes dragonfly as predator look for alternative food other than leafthoppers. Additional food is needed to keep adult predatory species from becoming extinct so that the balance between pest and both of predator species is controlled [2].

Mathematical models provide more insight into prey-predator population dynamics. Many researches have developed in different models of one prey-two predator or two prey-one predators $[3,4,5,6]$, and a stage-structure for predators $[7,8,9,10]$. Model [10] also discusses the Rosenzweig-MacAthur model with the effect refuge for immature prey. Tang and Xiao [11] has introduced anti-predator behaviour for predator. Panja [12] proposes effects anti-predator defense in a preypredator model using Ratio-dependent functional response to make the model more realistic. Prasad [13], Ulfa [14], and Zhu et al [15] incorporate food additional for predators in their model. Based on the background of several studies of prey-predator models, we developed a one prey-two predators' model to provide an overview of the dynamics of change through numerical simulations.

\subsection{Mathematical Model}

\subsubsection{Basic Model}

A one prey-two predator model will be constructed based on several assumptions. First, the predators consist of a stage-structure, namely juvenile predators and adult predators. The prey model with two predators considers the effects of addfood and anti-predator defenses for juvenile predators.

The level of predation of predators on prey affects changes in population growth. Holling [16] has acquainted functional responses that depend only on the prey species, namely Holling types I,II, and III. Savitri [17], has introduced a mathematical model with two predators using ratio-dependent functional response. Salamah, et.al [18] considered an anti-predator in a modified Leslie-Gower model with Beddington-

\footnotetext{
* Corresponding author :diansavitrir@unesa.ac.id
} 
DeAngelis functional response. Apriyani, et al [19] discussed ratio-dependent functional responses and also considered the effect of anti-predator defense in which prey can attack juvenile predators.

\subsubsection{Construction Model}

Based on predator-prey model proposed [17-19], we developed three models with different functional responses. In this paper we evolve of the prey population, juvenile predators, and adult predators model to delve the dynamics and also incoporates addfood and anti-predator defense. The model is obtained as follows

$$
\begin{gathered}
\frac{d x}{d t}=r x\left(1-\frac{x}{k}\right)-\frac{\alpha x y_{2}}{m+x+n A}, \\
\frac{d y_{1}}{d t}=\frac{\rho x y_{2}}{m+x+n A}-\beta y_{1}-\gamma x y_{1} \\
\frac{d y_{2}}{d t}=\beta y_{1}-\mu y_{2}
\end{gathered}
$$

Suppose $x$ is the population density of prey, $y_{1}$ represents of juvenile predator, and $y_{2}$ represents of adult predator the population density. All parameters relevant to the system (1) are positive and are described in Table 2. System (1) uses Holling type II functional response on predation rates of adult predators on prey.

\subsection{Material and methods}

We have studied local stability and system solutions and conceived of the existence of several equilibria points. Numerical simulations were performed using RungeKutta integrals of fourth-order which were displayed through phase portraits with Python 3.8 Software. To view the complete dynamics of the system, numerical continuity with MatCont is used, The parameters selected for continuity denote the effect of changing the stability of the equilibria point. Change in bifurcation dynamics showed by variations in add food parameter for predators, namely $A$.

\section{Result and Discuss}

\subsection{Equilibria}

The equilibria point is received from the equilibria solution of the system (1). The growth rates of each population is zero. The equilibria point describes the constant solution of the system. System (1) has four equilibrium points, as follows.

Table 1. The existence and type equilibria point.

\begin{tabular}{|c|l|}
\hline The equilibria & Type of equilibria \\
\hline$E_{0}=(0,0,0)$ & The extinction all population \\
\hline$E_{1}=(\mathrm{k}, 0,0)$ & $\begin{array}{l}\text { The extinction of both } \\
\text { predator, namely juvenile } \\
\text { predator and adult predator }\end{array}$ \\
\hline$E_{2}=\left(x^{2 *}, y_{1}^{2 *}, y_{2}^{2 *}\right)$ & The interior equilibria point \\
\hline$E_{3}=\left(x^{3 *}, y_{1}^{3 *}, y_{2}^{3 *},\right)$ & The interior equilibria point \\
\hline
\end{tabular}

We provide sufficient conditions for the existence of a nonnegative root. For $x^{*}$ we get equation.

$$
\omega_{1}\left(x^{*}\right)^{2}+\omega_{2}\left(x^{*}\right)+\omega_{3}=0
$$

With $\omega_{1}=\eta \mu$,

$$
\begin{aligned}
& \omega_{2}=A \eta n \mu+\eta \mathrm{m} \mu-\beta \rho+\beta \mu, \\
& \omega_{3}=A \beta n \mu+\beta m \mu
\end{aligned}
$$

In contrast to all of the equilibria point, $E_{2}=\left(x^{2 *}\right.$, $\left.y_{1}^{2 *}, y_{2}^{2 *}\right)$ and $E_{3}=\left(x^{3 *}, y_{1}^{3 *}, y_{2}^{3 *},\right)$ indicates that the prey, juvenile predators and adult predators can coexist.

\subsection{The Stability of Equilibria}

Point stability is executed by linearizing the system (1) using the Jacobi matrix [20].

The Jacobi matrix for equilibria point $E_{0}$

$$
J\left(E_{0}\right)=J(0,0,0)=\left[\begin{array}{ccc}
r & 0 & 0 \\
0 & -\beta & 0 \\
0 & \beta & -\mu
\end{array}\right] .
$$

Eigenvalue of the Jacobi matrix $J\left(E_{0}\right)$ are $\lambda_{1}=r>$ $0, \lambda_{2}=-\beta<0$, and $\lambda_{3}=-\mu<0$. Since there are positive eigenvalue, The equilibria point $E_{0}$ is also unstable, saddle point.

The Jacobi matrix for equilibria point $E_{1}$

$$
J\left(E_{1}\right)=J(k, 0,0)=\left[\begin{array}{ccc}
-r & 0 & -\frac{\alpha k}{A n+k+m} \\
0 & -\eta k-\beta & \frac{\rho k}{A n+k+m} \\
0 & \beta & -\mu
\end{array}\right] .
$$

With eigenvalue $\lambda_{1}=-r<0$, the other two eigenvalue $\lambda_{2}$ and $\lambda_{3}$ are determined by the quadratic equation $\lambda^{2}-T_{1} \lambda+D_{1}=0$. Because $T_{1}<0$ and $D_{1}>0$, then the eigenvalue for $\lambda_{2}, \lambda_{3}<0$ are met, The equilibria $E_{1}$ is locally asymptotically stable. This indicates that the juvenile predators and adult predators are extinct so there is only a prey population.

The stability of the equilibria point $E_{2}$ and $E_{3}$ will be asymptotically stable if certain conditions are fulfilled. It shows that all population, namely pre population, juvenile predators, and adult predators will coexist. Therefore, the extinction of juvenile predators and adult predators or extinction of all populations does not occur in the long term. We next investigated the stability of $E_{0}, E_{1}, E_{2}$, and $E_{3}$ by numerical simulation.

\section{Numerical Simulations}

The analytical solution of system (1) is not easy to decide. Therefore, numerical simulation can be performed to delve the system behavior. To see the behaviour of the solution as a whole, it is identified the local stability of equilibria point $E_{1}$ and $E_{2.3}$ by varying the value of the addfood parameter $(A)$ and deliniate change occured of bifurcation diagram by MatCont. Next, we provide simulation applying the parameter in Table 2, all of the equilibria exist. 
Table 2. Parameter model used for simulation.

\begin{tabular}{|c|c|c|}
\hline Parameter & Description & value \\
\hline$r$ & The intrinsic growth rate of prey & 6.6 \\
\hline$k$ & Carrying capacity of prey & 3.5 \\
\hline$\alpha$ & $\begin{array}{c}\text { The attack rate of adult predators on } \\
\text { prey }\end{array}$ & 1.6 \\
\hline$A$ & Addfood & $\begin{array}{c}2.416 \\
65\end{array}$ \\
\hline$n$ & $\begin{array}{l}\text { Relative ability of the both predator } \\
\text { to detect additional food to prey }\end{array}$ & 1.2 \\
\hline$m$ & $\begin{array}{l}\text { The coefficient of environmental } \\
\text { protection for the prey }\end{array}$ & 1.3 \\
\hline$\beta$ & $\begin{array}{l}\text { The growth rate of adult predators } \\
\text { comes from the transition of juvenile } \\
\text { predators into adult predator }\end{array}$ & 0.7 \\
\hline$\rho$ & $\begin{array}{l}\text { Proportionality constant, the } \\
\text { conversion efficiency of predation }\end{array}$ & 1.1 \\
\hline$\eta$ & Anti-predator & 0.3 \\
\hline$\mu$ & Natural death & 0.2 \\
\hline
\end{tabular}

\subsection{Bifurcation Diagram}

In numerical continuity, varying the value of the addfood parameter $(A)$ is to indicate changes in the stability of several the equilibria point. Figure 1 presents the bifurcation diagram of the solution of the system (1) which is varying the parameter addfood for both of the predator.

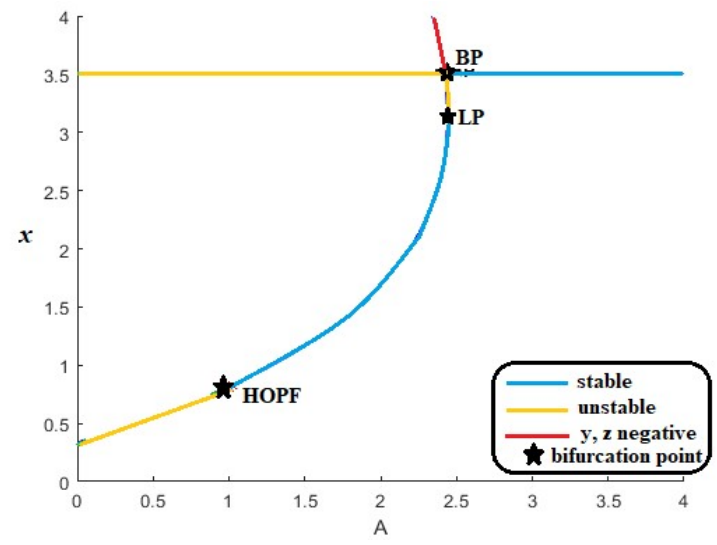

Fig. 1. Bifurcation Diagram of System (1).

a. Hopf Bifurcations

We perform numerical continuation by selecting the parameter of addfood for the predator which is $A$, the other parameter values remain constant from Table 2 . Therefore, the emergence of Hopf bifurcation is driven by addfood parameter for the predator $(A)$. The Hopf bifurcation point occurs when $A=1.00162435$ as shown in Figure 2. The interior equilibria point for $E_{2}=$ $(0.812441,2.999504,10.498265)$ when the Hopf bifurcation occurs.

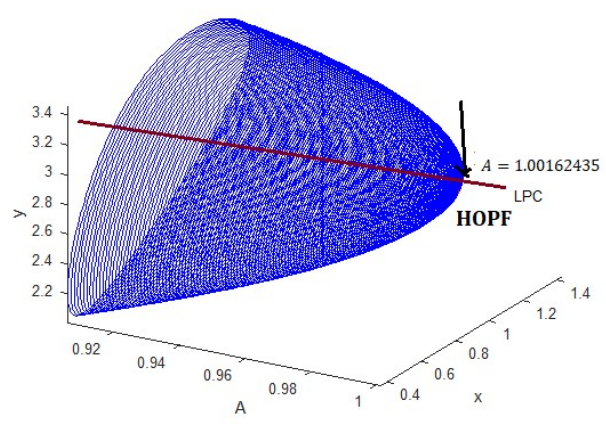

Fig. 2. Hopf Bifurcation of System (1) in equilibria point $\boldsymbol{E}_{2}$ at $\boldsymbol{A}=\mathbf{1 . 0 0 1 6 2 4 3}$

a. Saddle-node phenomenon

The results of the numerical continuation of the parameter $\boldsymbol{A}$ show the changes that occur in the stability behavior at the interior equilibria point $\left(\boldsymbol{E}_{2}\right)$. The Bifurcation diagram in Figure 1 shows for 2. $416667<$ $\boldsymbol{A}<2 . .435303$. There are two interior equilibria points where the difference in stability is $\left(\boldsymbol{E}_{2}\right)$ and $\left(\boldsymbol{E}_{\mathbf{3}}\right)$. The phenomenon of the emergence of LP namely Limit Point shows that there is a Saddle-node (Fold) bifurcation which is driven by the parameter of addfood for the predator.

\subsection{Phase Portraits}

The dynamics of the solution of the system (1) have been observed in Figure $4-6$ of the above by phase portrait. The figure 3 show the dynamic solution of the system (1).

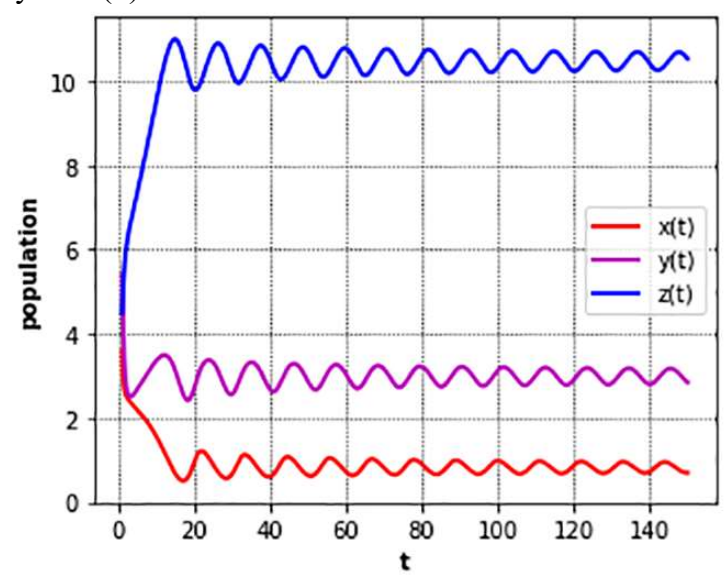

Fig. 3. Timeseries of System (1).

Based on the paremeter value in Table 2 and choose addfood parameter $A=1.00162435$, the equilibria point $E_{0}, E_{1}$, and $E_{2}$ are exist. The value of each point of equilibria are $E_{0}=(0,0,0), E_{1}=(3.5,0,0)$, and $E_{2}=$ (0.812441, 2.999504, 10.498265). Therefore, $E_{0}, E_{1}$ and $E_{3}$ are unstable and $E_{2}$ is asymptotically stable. 


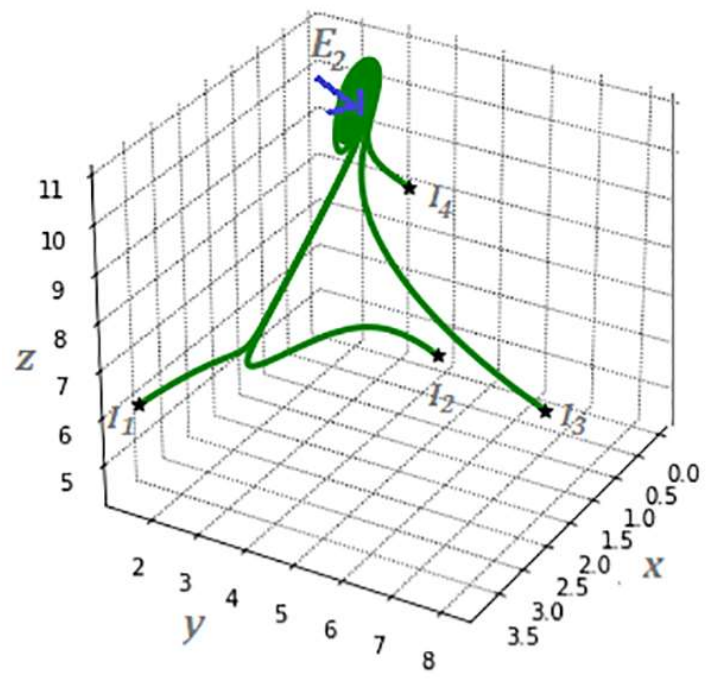

Fig. 4. The Phase Portrait of System (1) leads to the

\subsection{Interior equilibria point for $E_{2}$ at $A=$ 1. 00162435.}

The initial value in Figure 4 shows the initial density of all population. With initial value $I_{1}[3.8 ; 1.5 ; 6.2]$, $I_{2}[0.1 ; 3.9 ; 4.6], I_{3}[2.1 ; 8.1 ; 6.5], I_{4}[1.1 ; 4.3 ; 9.2]$. For different initial value, all numerical solution of system (1) convergen to the interior equilibria point $\left(E_{2}\right)$, and $E_{2}$ is an asymptotically stable. The result are shown in Figure 4.

We use initial value $I_{1}[3.8 ; 1.5 ; 6.2]$, $I_{2}[2.1 ; 8.1 ; 6.5], I_{3}[1.1 ; 4.3 ; 7.2]$, all numerical solution of system (1) lets to the extinction of the juvenile predator and adult predator. The equilibria point $\left(E_{1}\right)$ is an asymptotically stable. The eigen value of $J\left(E_{1}\right)$ are $-3.9985 \times 10^{-9}+0.5756 I,-3.9985 \times$ $10^{-9}-0.5756 I$, and -1.4335 .

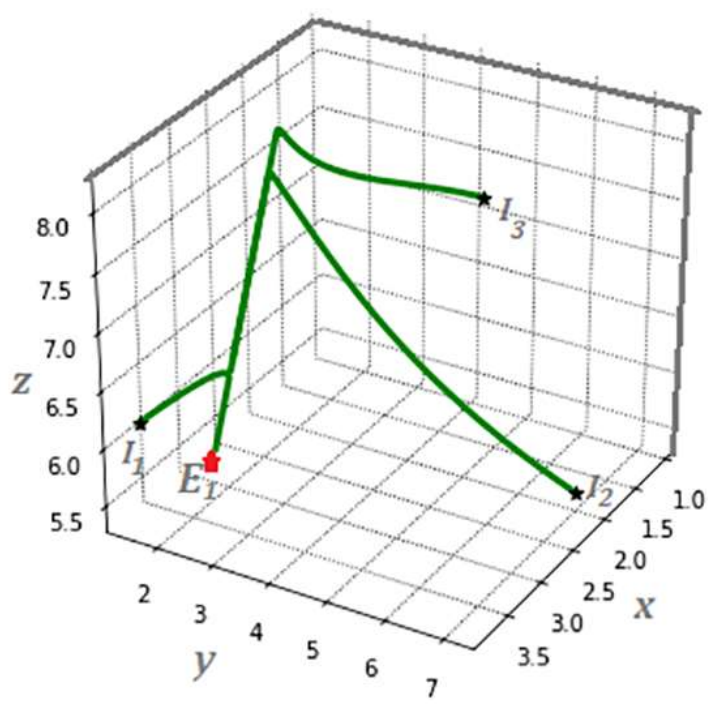

Fig. 5. The Phase Portraiet of System (1) in equilibria point $\boldsymbol{E}_{\mathbf{1}}$ at $\boldsymbol{A}=\mathbf{1 . 0 0 1 6 2 4 3}$

\subsection{Double stability phenomenon.}

Another interesting dynamics behavior to observe as shown in Figure 5 is the appearance of two different stability of the equilibrium points, known as the bistability phenomenon. This phenomenon occurs at $A=$ 1.00162435. There are two stabilities in the solution of system (3) namely the interior equilibria point $\left(E_{2}\right)$ and the juvenile predator and adult predator extinction equilibria point $\left(E_{1}\right)$ which are locally asymptotically stable. This phenomenon shows that the system (3) has a double stability

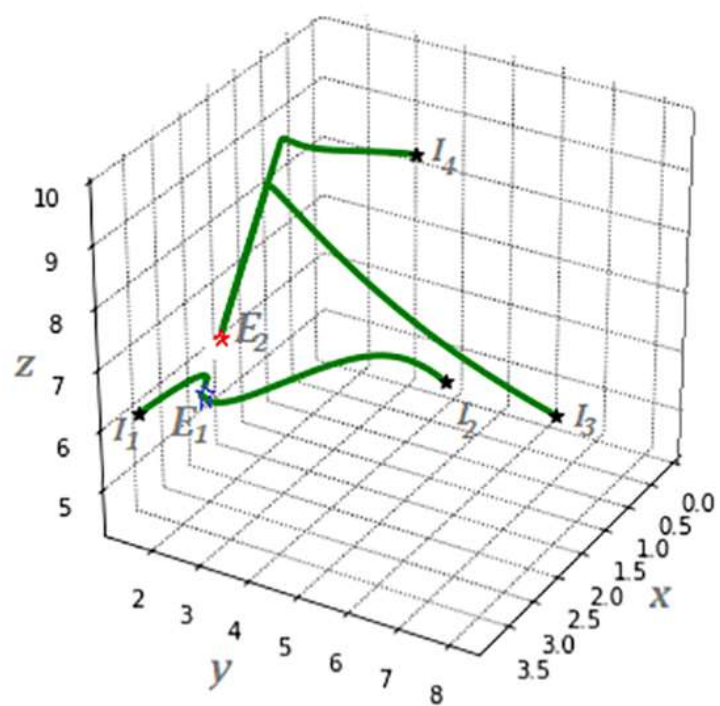

Fig. 6. The Phase Portriet of System (1) in equilibria point $\boldsymbol{E}_{1}$ and $\boldsymbol{E}_{2}$ at $\boldsymbol{A}=\mathbf{1 . 0 0 1 6 2 4 3}$

The equilibria point $E_{0}=(0,0,0), E_{1}=(3.5,0,0)$, and $E_{2}=(2.8,1.6,5.7)$ are exist. We use the same initial value with $I_{1}[3.8 ; 1.5 ; 6.2]$, $I_{2}[0.1 ; 3.9 ; 4.6], I_{3}[2.1 ; 8.1 ; 6.5]$, and $I_{4}[1.1 ; 4.3 ; 9.2]$. The numerical solution of system (1) lets to the extinction of the juvenile predator and adult predator $\left(E_{1}\right)$ for initial value $I_{1}$ and $I_{2}$. The equilibria point $\left(E_{1}\right)$ is an asymptotically stable. The eigen value of $J\left(E_{1}\right)$ are $-6.6,-1.9$, and $-9.3 \times 10^{-9}$. Initial value $I_{3}$ and $I_{4}$ convergen to $E_{2}=(2.8,1.6,5.7)$. The interior equilibria point $\left(E_{2}\right)$ is asymptotically stable. The eigen value of $J\left(E_{2}\right)$ are $-4.7,-1.7$, and -0.002 .

This study shows the addfood for predator has a meaningful on the dynamics of the prey population, juvenile predators and adult predators population. Therefore, it is prime for ecological models to combine predation affect both of the population interaction and additionalfood for predator. However, another possibilityis to maintain the ecology at coexistence working if possible uses the other parameter.

Finally, we notice that the all population can live together with some conditions described. The addfood of the predator helps two populations to survive for a long period of time without extinct. 


\section{Conclussion}

1. We have numerically studied the population models of preys, juvenile predator, and adult predators model with Holling Type II functional response by considering addfood and anti-predator defense. The model has four equilibria points, namely the trivial point $\left(E_{0}\right)$, the extinction point both of the predator $\left(E_{1}\right)$, and the survival of prey, juvenile predators, and adult predators point $\left(E_{2}\right.$ and $\left.E_{3}\right)$ are stable under certain conditions. Increasing the parameter addfood for both of predators (juvenile and adult) may stabilize equilibria point $\left(E_{1}\right)$. It is can prevent extinction on the population of the juvenile predator.

\section{References}

1. Molles MC. Ecology Concept and Applications. 2nd Edition, Mexico City: McGraw Hill, (2002).

2. Campbell NA, ReeceJB, MitchellLG. Biologi Jilid3.Edisi 5. Jakarta : Erlangga, (2004).

3. Sarwardi S and Mandal PK, Dynamical behaviour of a two-predator model with prey refuge $J$. Bio Phys 39 : 701-722, (2013).

4. Khajanchi S, Dynamic behavior of a BeddingtonDeAngelis type stage structure predator-prey model, J. App. Math. And Comp., 244 344-360, (2014).

5. Savitri D and Abadi, Numerical Simulation in Prey-Predator Model with a structure for prey Atlantis Highlights in Engineering (AHE), (2018).

6. L. K. Beay, A. Suryanto, I. Darti, and Trisilowati, "Stability of a stage-structure RosenzweigMacArthur model incorporating Holling type-II functional response," IOP Conference Series: Materials Science and Engineering, vol. 546, p. 052017, (2019).

7. Savitri D, Stability and Numerical Simulation of Prey-predator System with Holling Type-II Functional Responses for Adult Prey, J. of Phys. : Conference Series, vol : 1417 issue : 1-12-20, 1417 012025, (2019).

8. Falconi, Huenchucona M, and Vidal C, Stability and global dynamic of stage-structured predator-prey model with group defense mechanism of the prey J. App. Math. And Comp 270 47-61, (2015).

9. S. Khajanchi and S. Banerjee, "Role of constant prey refuge on stage structure predator-prey model with ratio dependent functional response," Applied Mathematics and Computation, vol. 314, pp. 193198, (2017).

10. Beay LK, and Saija M, A Stage-Structure Rosenzweig-MacArthur Model with Effect of Prey Refuge, Jambura J. Biomath, Vol. 1, Issue 1: June, (2020).

11. Tang B and Xiao Y, Bifurcation analysis of a predator-prey model with anti predator behaviour, Chaos, Solitons \& Fractals 70, 58-68, (2015).

12. Panja $\mathrm{P}$, Mondal $\mathrm{S}$ and Chattopadhyay J, Dynamical Effects of Anti-predator of Adult Prey in a Pretator-prey Model with Ratio-dependent Fuctional Response, Asian J. of Math and Phys, Vol. 1. ISSUE I, 2017, 19-32, (2017).

13. B. S. R. V. Prasad, M. Banerjee, P. D. N. Srinivasu, Dynamics of Additional Food Provided PredatorPrey System with Mutually Interfering Predators, Mathematical Biosciences, 176-190, (2013).

14. H.M. Ulfa, A. Suryanto, and I. Darti, Dynamics of Leslie-Gower Predator-prey Model with additional food for Predator, J. of Pure and Appl. Math., vol. 115, no. 2, p. 199-209, (2017).

15. Zhu et al., The Influence of fear effect to the LotkaVolterra predator-prey System with Predator has other food Resource., Advances in Difference Equations., https://doi.org/10.1186/s13662-02002612-1, (2020).

16. Holling CS. The Functional Response of Predator to Prey Density and its Role in Mimicry and Population Regulation.Memoirs of the Entomological Society of Canada1965;97: 5-60.

17. Savitri D, Dynamics Analysis of Anti-Predator Model on Intermediate Predator With Ratio Dependent Functional Responses, IOP Conf. Series: Journal of Physics: Conf. Series 953 doi :10.1088/1742-6596/953/1/012201, (2018).

18. Salamah U, Suryanto A, and Kusumawinahyu, W.M., AIP Conf. Proc. 2084, 020001-1-0200017; https://doi.org/10.1063/1.5094265, (2019).

19. Apriyani. A, Darti. I., and Suryanto. A, AIP Conference Proceedings 2084, 020002; https://doi.org/10.1063/1.5094266, (2019).

20. Boyce, W. E., \& DiPrima, R. C., Elementary differential equations and boundary value problems. xvi+809, (2012). 